\title{
Buchbesprechungcn - Book Reviews - Livres nouveaux
}

Adolf Roos: Die Kriegsernährung in ihrer Bedeutung für die Entwicklung der Zahn· karies in der Schweiz. Buchdruckerei Berichthaus Zurich, 1950. 207 Seiten.

Der Altmeister der Kariesforschung in der Schweiz, Dr. med. Adolf Roos, hat in seinem Buch«Die Kriegsernährung in ihrer Bedeutung für die Entwicklung der Zahn-karies in der Schweiz » einen sehr wertvollen Beitrag zum Kariesproblem geliefert. In diesem Buch, das der Autor eine kritische Vergleichsstudie nennt, finden wir sta-tistische Angaben aus dem Krankengut der städtischen Schulzahnkliniken von Basel, Zurich, St. Gallen, Winterthur, Bern, Bischofszell und anderen. Durch die Zusammenarbeit mit dem Statistischen Amt des Kantons Basel-Stadt ist gewähr-leistet, daß die Auswertung auch in statistischer Hinsicht einwandfrei ist. Wir haben es daher mit einem Werk zu tun, das auf Grund des, man ist versucht zu sagen einmaligen, Materials sehr bald zu einem Standardwerk der Kariesforschung werden wird. Dieses Buch ist um so wertvoller, als es von einem Mann geschrieben worden ist, dessen Bedeutung und Pionierarbeit für die Kariesforschung überall bekannt ist. Es ist deshalb besonders wertvoll, weil es sich nicht nur mit der Kariesstatistik in den Jahren 1934-1948 beschäftigt, sondern weil auch ein besonderes Kapitel der Frage der Beziehung zwischen Ernährung und Karies gewidmet ist. Von beson-derem Interesse ist der Teil «Vergleich der eigenen Untersuchungen mit den Ergeb-nissen in anderen Ländern». Das Buch zeigt mit der gleichen Deutlichkeit wie die Arbeiten von Toverud in Norwegen, welche dominierende Rolle der Rohrzucker und die aus ihm hergestellten Süßwaren in der Kariesätiologie spielen. Bei aller Wichtig-keit dieser Erkenntnisse ist es jedoch nicht angebracht, den Nährwert des Rohr-zuckers leugnen zu wollen. Die in dem Buch gelegentlich angedeutete Auffassung, die in den einschlägigen Industrien verarbeiteten Nahrungsmittel seien insgesamt minderwertig, entspricht nicht den modernen Untersuchungen. Jeder Ernährungs-physiologe ist sich dessen bewußt, daß eine Verarbeitung der Nahrungsmittel unter den heutigen Zivilisationsbedingungen eine unbedingte Notwendigkeit ist und daß gewisse Nährwertverluste in Kauf genommen werden müssen. Nach Meinung des Referenten ist es aber nicht angängig, den «lndustriezucker», das heißt den verarbeiteten Rohzucker, als ein minderwertiges Nahrungsmittel hinzustellen. Der Referent ist sich bewußt, unter anderem auf Grund eigener experimenteller Arbeiten, welche zentrale Bedeutung gerade dem Zucker und den Süßwaren in der Kariesentstehung zukommt, glaubt jedoch darauf aufmerksam machen zu müssen, daß der Zucker als Energielieferant ein bedeutendes Nahrungsmittel darstellt. Auch kann der Ansicht des Verfassers nicht immer Folge geleistet werden, wenn zum Bei-spiel erwähnt wird (Seite 164 oben), daß der Fruchtzucker wertvoller sei als der Rohrzucker. Die Bezeichnung «naturreiner, edler Fruchtzucker» geht über die rein sachliche wissenschaftliche Beurteilung des Zuckers hinaus. Es ist außerdem experi-mentell nachgewiesen worden, daß Fruchtzucker sehr kariogen ist und wenn er im Tierversuch im Vergleich zum Rohrzucker geprüft wird, durchaus stark kariogen sein kann. Unbedingt zuzustimmen ist dagegen dem Verfasser, wenn er auf die große gesundheitliche und ernährungsphysiologische Bedeutung der Obstsäfte hinweist. Sehr wertvoll ist vor allem die Tatsache, daß Roos beachtliche Vorschläge gemacht hat, das Süßigkeitsbedürfnis, vor allem der Kinder, durch Obst zu decken. Mit 
Ausnahme dieser oben erwähnten Tatsachen vermag der Referent dem Verfasser vollauf zu folgen in seinen Schlußfolgerungen über den engen Zusammenhang zwischen Ernährung und Karies. Der Referent glaubt aber darauf hinweisen zu müssen, daß die kariesprophylaktische Wirkung des mineralreicheren Schwarz- und Vollkornbrotes häufig sehr überschätzt wird. Sämtliche Untersuchungen über den

80 Buchbesprechungen - Book Reviews - Livres nouveaux

Zusammenhang zwischen Kriegskost und Kariesfrequenz haben ergeben, genau wie auch die Untersuchungen von MacGregor in Birmingham im letzten Jahr in Ghana, daß die ärmeren Bevòlkerungsteile, die ernährungsphysiologisch gesehen gròßten-teils unzureichend erkannt werden, den geringsten Kariesprozentsatz aufwiesen. Diese immer wieder bestätigte Tatsache gibt zu denken und läßt uns den Begriff «vollwertige Nahrung»mitVorsichtverwenden. Es darf nicht vergessen werden, daß die Kriegskost in der Schweiz ernährungsphysiologisch gesehen wesentlich besser war als beispielsweise in manchen besetzten Ländern während des Krieges und in Deutschland in den Jahren unmittelbar nach dem Kriege.

Im ganzen gesehen müssen wir Roos dankhar sein, daß er die schwierige Aufgabe auf sich genommen hat, uns das äußerst wertvolle Material in einer Form vorzulegen, an der niemand vorbeigehen kann, der sich ernsthaft mit der Kariesforschung beschäftigt. Man kann dem Werk nur eine weite Verbreitung wünschen und hoffen, daß es auch in andere Sprachen übersetzt wird. Fritz Bramstedt, Hamburg Standard Methods of the Oils and Fats Division of the Y.U.P.AC. 6th edition, Butter-worths, London 1964. Prix: $50 \mathrm{~s}$.

La section des matières grasses de $\Gamma$ Union Internationale de Chimie pure et appliquée présente la 6e edition de ses fiches d'analyses.

La premiere section a trait aux analyses de graines et fruits oléagineux: dosage des impuretés, broyage, dosage de l'humidité, de Thuile, de Гacidité.

La deuxième section traite de Tanalyse des matières grasses: preparation des échantillons, indice de refraction, couleurs, dosage des substances étrangères, des caractéristiques chimiques, indices d'acides de saponification, d'esters, de l'in-saponifiable des stérols, des peroxydes, etc.

Les textes sont en français et en anglais.

II s'agit d'un manuel de reference pour tout laboratoire ayant à caractériser des

matières grasses. J. Trémolíères, Paris

Herbert Krauss: Gesunde Küche. VEB Verlag Volk und Gesundheit, Berlin 1965. 295 S., 8 Abb. Preis: DM 10.-.

Das Buch gibt eine Übersicht über die wichtigsten diätetischen Probleme und hat in alphabetischer Reihenfolge eine Nahrungsmittelkunde, die allerdings nicht den Anspruch auf Vollständigkeit erhebt. Diese Nahrungsmittelkunde ist aber sicherlich sehr praktisch. Das Kapitel «Wie kaufe ich ein?» wird vielleicht im Westen weniger Bedeutung haben. Die Veränderungen der Nahrungsmittel, die im nächsten Kapitel besprochen werden, decken sich mit den üblichen Auffassungen. Die Speisepläne, die beigegeben sind, haben für uns nur wenig Bedeutung, ebenso die meisten Kochrezepte.

Alles in allem ein Buch, das trotz ausgezeichneter Qualitäten sich im Westen nicht recht durchsetzen wird, obwohl gewiße Kapitel, besonders aus der Nahrungsmittelkunde (Fleischwaren und Benennung der Fleischarten, Beschreibung der Küchengewürze und Ursprung derselben) in den üblichen Diätbüchern nicht ange-führt werden und Nachahmung verdienen.

Das Buch ist gut ausgestattet und im Verhältnis dazu mit DM 10.- ausgespro-

chen billig. R. Boiler, Wien 